\title{
RNA-Seq transcriptome reveals different molecular responses during human and mouse oocyte maturation and fertilization
}

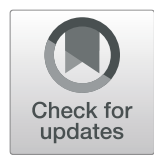

Zheng-Hui Zhao ${ }^{1,2}$, Tie-Gang Meng ${ }^{1,3}$, Ang Li ${ }^{1}$, Heide Schatten ${ }^{4}$, Zhen-Bo Wang ${ }^{1,2^{*}}$ and Qing-Yuan Sun ${ }^{1,3^{*}}$ (D)

\begin{abstract}
Background: Female infertility is a worldwide concern and the etiology of infertility has not been thoroughly demonstrated. Although the mouse is a good model system to perform functional studies, the differences between mouse and human also need to be considered. The objective of this study is to elucidate the different molecular mechanisms underlying oocyte maturation and fertilization between human and mouse.

Results: A comparative transcriptome analysis was performed to identify the differentially expressed genes and associated biological processes between human and mouse oocytes. In total, 8513 common genes, as well as 15, 165 and 6126 uniquely expressed genes were detected in human and mouse MII oocytes, respectively. Additionally, the ratios of non-homologous genes in human and mouse MII oocytes were 37 and 8\%, respectively. Functional categorization analysis of the human MII non-homologous genes revealed that CAMP-mediated signaling, sister chromatid cohesin, and cell recognition were the major enriched biological processes. Interestingly, we couldn't detect any GO categories in mouse non-homologous genes.

Conclusions: This study demonstrates that human and mouse oocytes exhibit significant differences in gene expression profiles during oocyte maturation, which probably deciphers the differential molecular responses to oocyte maturation and fertilization. The significant differences between human and mouse oocytes limit the generalizations from mouse to human oocyte maturation. Knowledge about the limitations of animal models is crucial when exploring a complex process such as human oocyte maturation and fertilization.
\end{abstract}

Keywords: Oocyte maturation, Transcriptome, Fertilization, Transcripts degradation

\section{Background}

Ovarian folliculogenesis is an extremely species-specific process and the formation of a mature oocyte starting from a primordial follicle is completed in several weeks in mice [1], but several months in human [2]. Although certain molecular mechanisms underlying fundamental functions of oocyte maturation should be conserved among gamogenetic species [3], the differences between species in oocyte maturation need to be considered. For

\footnotetext{
*Correspondence: wangzb@ioz.ac.cn; sunqy@ioz.ac.cn

${ }^{1}$ State Key Laboratory of Stem Cell and Reproductive Biology, Institute of Zoology, Chinese Academy of Sciences, Beijing, China

Full list of author information is available at the end of the article
}

instance, protein synthesis is essential for germinal vesicle breakdown (GVBD) in human [4] but not in mice [5]. Similarly, increased level of cyclic adenosine monophosphate (cAMP) or cyclic guanosine monophosphate (cGMP) promotes the GVBD in nemertean oocytes, but instead significantly blocks the GVBD in mammals [6]. In addition, physiological concentrations of glucorticoids do not affect mouse oocyte maturation, but typically inhibit the nuclear maturation of pig oocytes [7]. The nonhomologous genes that are different among species may contribute to these species-specific molecular pathways.

Fusion of sperm and oocyte is a common aspect that initiates embryo development in gamogenetic species. 
The transition from oocyte to embryo mainly relies on maternal RNAs and proteins that are generated during oocyte growth [8]. Fully-grown GV oocytes are transcriptionally silent before meiosis resumption until zygote genome activation occurs after fertilization [9-11]. The necessary transcripts deposited in fully-grown GV oocytes are produced during the period of oocyte growth, which is essential for oocyte maturation and fertilization [12]. Also, the selective degradation of transcripts that occurs during oocyte maturation is required for meiotic maturation and oocyte-to-embryo transition [13-15]. Although human and mouse oocytes undergo degradation of maternal mRNAs during oocyte maturation, the time period of oocyte to embryo transition is different between human and mouse.

Several studies have compared the microarray data [16-18], however the differences in oocyte maturation and fertilization between human and mouse have not been fully characterized. Here, we compared the RNASeq data of human and mouse oocytes during the transition from the GV stage to the MII stage. As a result, 2243 and 2488 transcripts are completely degraded during oocyte maturation in mouse and human, respectively. Compared to the GV oocytes, 430 and 3790 transcripts appear exclusively in mouse and human MII oocytes, respectively. Moreover, the ratio of nonhomologous genes is significantly different between human and mouse oocytes. Collectively, these data suggest that the shared and exclusively expressed transcripts would discriminate between common and speciesspecific molecular mechanisms that regulate oocyte maturation and fertilization.

\section{Results}

\section{Slight differences in molecular features between mouse} GV and MII oocytes

Differences in gene expression profiles between mouse GV and MII oocytes were analyzed to determine the significantly changed transcripts that may contribute to meiotic maturation and fertilization processes. As expected, several transcripts that enriched in meiosis I cell cycle process degraded strikingly during GV to MII transition (Table 1). In the present study, the number of genes that are uniquely expressed in mouse GV and MII oocytes were 2243 and 430, respectively (Additional files 1 and 2). And 14,209 genes were co-expressed at both GV and MII stages (Fig. 1a). During the GV to MII transition, 2243 transcripts were selectively completely degraded. Of particular note, 430 transcripts appeared to be expressed uniquely in MII oocytes.

To ascertain differences in gene expression profiles between GV and MII oocytes, we used Clusterprofiler R package to analyze gene ontology of genes that are exclusively expressed in GV and MII oocytes [19]. As
Table 1 Degradation of transcripts during mouse oocyte GV-toMII transition

\begin{tabular}{|c|c|c|}
\hline Gene & GV FPKM & MII FPKM \\
\hline \multicolumn{3}{|c|}{ Meiosis I cell cycle process } \\
\hline Ccdc155 & 36.7227 & 1.45956 \\
\hline Cntdl & 2.49518 & 0.967175 \\
\hline Cep63 & 11.3733 & 1.94341 \\
\hline Mlh1 & 11.4869 & 0.343089 \\
\hline Top2b & 2.62691 & 1.2193 \\
\hline $\operatorname{cdc} 25 \mathrm{c}$ & 9.49562 & 0.884645 \\
\hline Syde1 & 3.37878 & 0.42659 \\
\hline Psmd13 & 2.53468 & 0.383028 \\
\hline Ercc1 & 12.7466 & 1.00127 \\
\hline Topbp 1 & 7.54961 & 1.90707 \\
\hline Eme2 & 4.15232 & 0.297086 \\
\hline \multicolumn{3}{|c|}{ Mitochondrion organization } \\
\hline Coa4 & 11.9735 & 1.01023 \\
\hline Tomm40 & 26.1551 & 0.763054 \\
\hline Hsd17b10 & 11.0287 & 0.491451 \\
\hline $\operatorname{Prd} \times 3$ & 20.9225 & 1.37221 \\
\hline $\operatorname{Cox} 16$ & 27.8022 & 0.713859 \\
\hline Mtch2 & 2.45931 & 0.200191 \\
\hline Mrp/15 & 7.85712 & 0.458739 \\
\hline Wdr45 & 3.63553 & 0.667861 \\
\hline $\operatorname{Trp} 73$ & 5.74592 & 1.35138 \\
\hline \multicolumn{3}{|c|}{ Cytoplasmic translation } \\
\hline Rp/24 & 5.34353 & 0 \\
\hline Dph5 & 4.02856 & 0.381827 \\
\hline Eif4h & 105.217 & 1.32238 \\
\hline Mcts2 & 2.54326 & 0 \\
\hline Rp/30 & 43.2177 & 1.20127 \\
\hline $\operatorname{Rps} 29$ & 9.1687 & 0 \\
\hline \multicolumn{3}{|c|}{ RNA modification } \\
\hline Qtrt1 & 7.28196 & 0.177408 \\
\hline Alkbh3 & 5.09442 & 0.907235 \\
\hline Aars2 & 4.09517 & 0.199085 \\
\hline Lcmt2 & 6.43164 & 0.932506 \\
\hline Nsun5 & 5.13521 & 1.89229 \\
\hline Ctu2 & 4.34528 & 0.460726 \\
\hline
\end{tabular}

shown in Additional files 3 and 4, of the 335 biological processes (BP), 239 are significantly correlated with the transcripts that are degraded during the GV to MII transition, whereas 96 are closely associated with the transcripts that appeared in MII oocytes. We compared the GO categories between the two groups, and found that several biological functions are similar in these two groups, such as "mononuclear cell proliferation", 


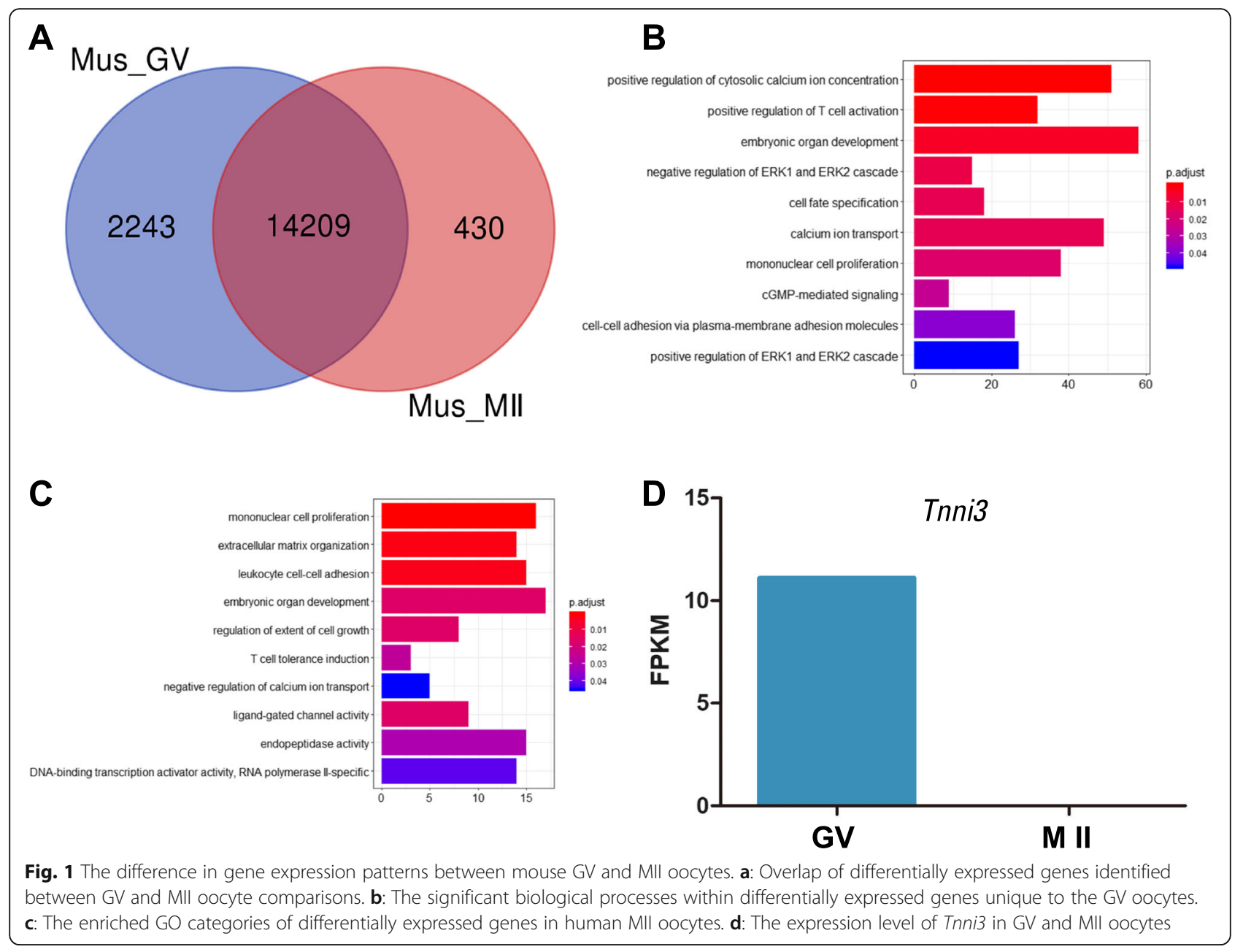

"embryonic organ development", "cell-cell adhesion" and "T cell functions" (Fig. 1b and c). Moreover, most of the biological processes are closely related. For instance, 41 transcripts are enriched both in "leukocyte cell-cell adhesion" and "regulation of $\mathrm{T}$ cell activation" (Additional file 3), which suggests that immunity-related factors may play essential roles in cell adhesion. In addition, 9 transcripts exclusively expressed in the GV stage are involved in the cGMP-mediated signaling pathway (Additional file 3), which indicates that these transcripts may maintain meiotic arrest in fully-grown GV oocytes. On the other hand, many genes expressed in the GV oocytes were enriched in calcium ion transport. For example, Tnni3 is a critical component in the calcium ion regulatory system, which is involved in developmental regulations [20]. Of particular note, the expression level of Tnni3 decreases significantly during the transition from GV to M II oocytes (Fig. 1d). Moreover, several genes involved in negative regulation of calcium ion transport were enriched in MII oocytes, indicating that the calcium ion transport process is active in fullygrown GV oocytes and that calcium ion homeostasis may be differentially regulated in GV oocytes and MII oocytes. The GO terms indicate that newly appearing genes in MII oocytes may play an important role in oocyte maturation and fertilization.

\section{Specific gene expression patterns in oocytes before and after fertilization}

Mammalian fertilization requires recognition, interaction and fusion between sperm and the mature oocyte, which subsequently initiates embryo development through zygote genome activation [21]. To further determine the dynamic changes and roles of genes specifically expressed in MII oocytes, we analyzed the transcriptomes of MII oocytes, zygotes and two-cell embryos (GSE71434) [22]. The ADAM and CD gene families are essential for fertilization [23]. However, we could not detect the expression of Adam2, $C d 46$ and $C d 79 a$ in the zygote stage, which indicates that these genes may play roles in the recognition and fusion stage between sperm and the oocyte (Fig. 2a and b). In addition, calcium oscillations are critical during fertilization, which triggers oocyte activation and cell cycle resumption [24]. The expression level 

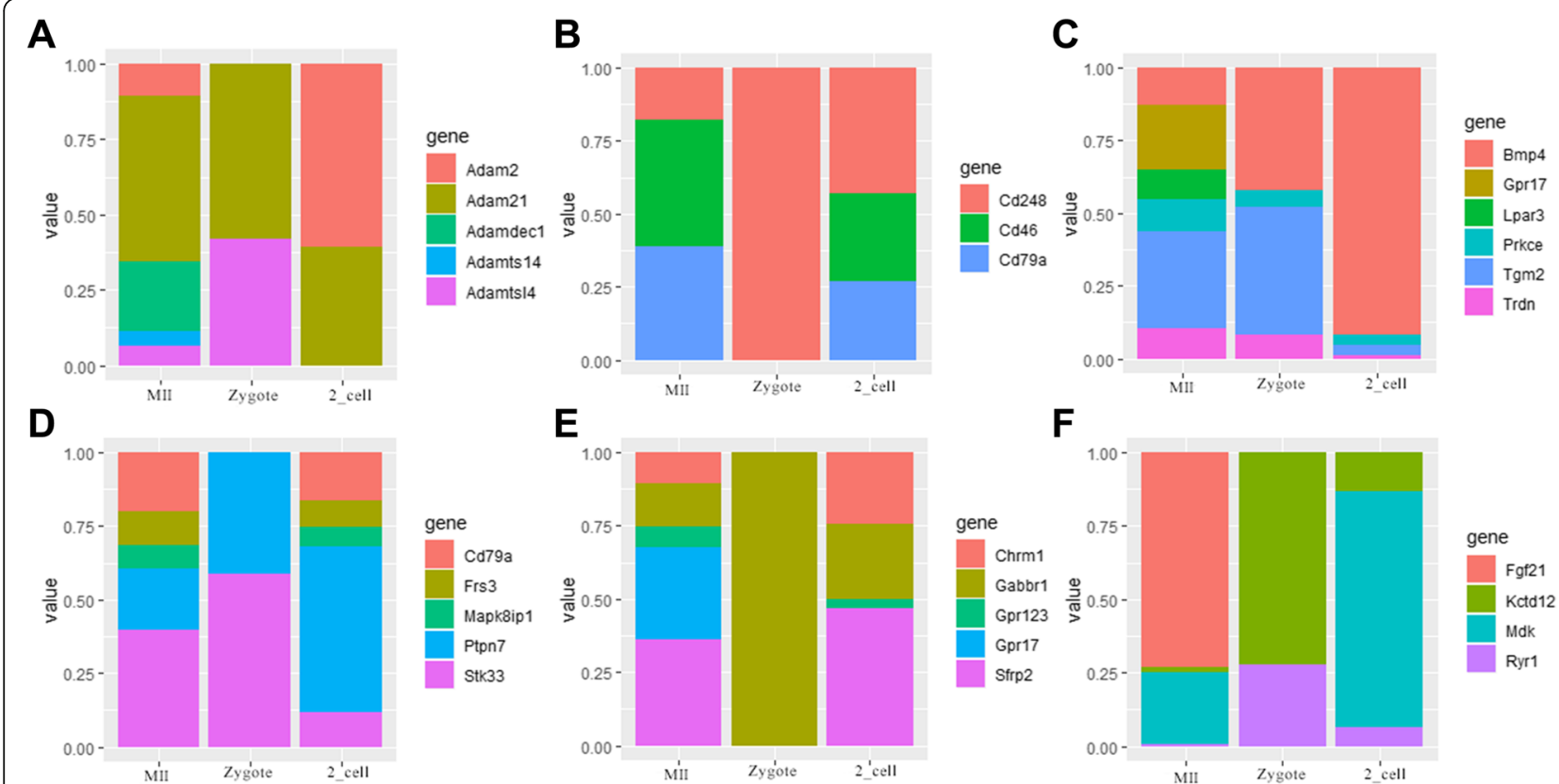

Fig. 2 Dynamic changes in gene expression in eggs around fertilization. a: Relative expression of Adam gene family. b: Relative expression of Cd gene family. c: Relative expression of $\mathrm{Ca}^{2+}$ homeostasis-related genes. $\mathbf{d}$ : Relative expression of MAPK signaling pathway-related genes. e: Relative expression of $\mathrm{G}$ protein-coupled peptide receptor activity-related genes. f: Relative expression of CAMP-PKA pathway-related genes

of Bmp4 increases during fertilization (Fig. 2c), which may cooperate with $\mathrm{Ca}^{2+}$ to regulate the SMAD1/5 signaling pathway [25]. Moreover, several signaling pathways are critical for fertilization; the Ptpn 7 and Stk33 that are enriched in mitogen-activated protein kinase (MAPK) pathway and Gabbr1 that is involved in G protein-coupled receptor pathway play important roles in the oocyte-to-embryo transition (Fig. $2 \mathrm{~d}$ and e). In the cAMP-PKA pathway, there is a decreased expression of Fgf21 and increased expression of Kctd12 during fertilization (Fig. 2f). Collectively, these data suggest that the genes that are exclusively expressed in MII oocytes may play important roles in fertilization.

\section{Significant differences in molecular features between human GV and MII oocytes}

To explore the molecular mechanisms in human oocyte maturation, we compared the gene expression profiles between human GV and MII oocytes. The numbers of genes that were exclusively expressed in human GV and MII oocytes were 2488 and 3790, respectively (Additional files 5 and 6). A total of 19,889 genes were co-expressed in both GV and MII stages (Fig. 3a). During the GV-to-MII transition, 2488 transcripts were selectively completely degraded. Additionally, the transcripts that enriched in mitochondrial translational termination and cytosolic transport degraded dramatically in the process of oocyte maturation (Table 2). In contrast to mouse MII oocytes, there were 3790 transcripts exclusively expressed in MII oocytes.

To further determine the features of human GV and MII oocytes, we performed GO and KEGG analysis of the genes that are uniquely expressed in human GV and MII oocytes. The gene ontology analysis revealed 257 categories, of which 217 are closely associated with the transcripts that are lost during oocyte maturation, whereas 40 are the transcripts that appear in MII oocytes (Additional files 7 and 8). Also, 34 KEGG pathways were detected among stage exclusively expressed transcripts (Additional files 9 and 10). Furthermore, we compared the GO terms and KEGG pathways between the two groups, and there were several similar categories such as "mononuclear cell proliferation", "cell-cell adhesion", "Retinol metabolism" and "cytokine-cytokine receptor interaction" (Fig. 3b-e). On the other hand, 16 transcripts that were exclusively expressed in the GV stage were enriched in the category of response to cAMP (Fig. 3b; Additional file 7), which suggests that these transcripts could participate in the regulation of meiotic arrest in fully-grown GV oocytes [26]. In addition, the term of positive regulation of cell adhesion mediated by integrin suggests that genes, such as CXCL13, SKAP1 and FOXC2, specifically expressed in MII stage may play an important role in oocyte maturation and fertilization (Fig. 3d; Additional file 8). Additionally, growth arrest and DNA damage 45G (GADD45G) is a reproduction related gene that is enriched in MAPK activities [27-29]. The 


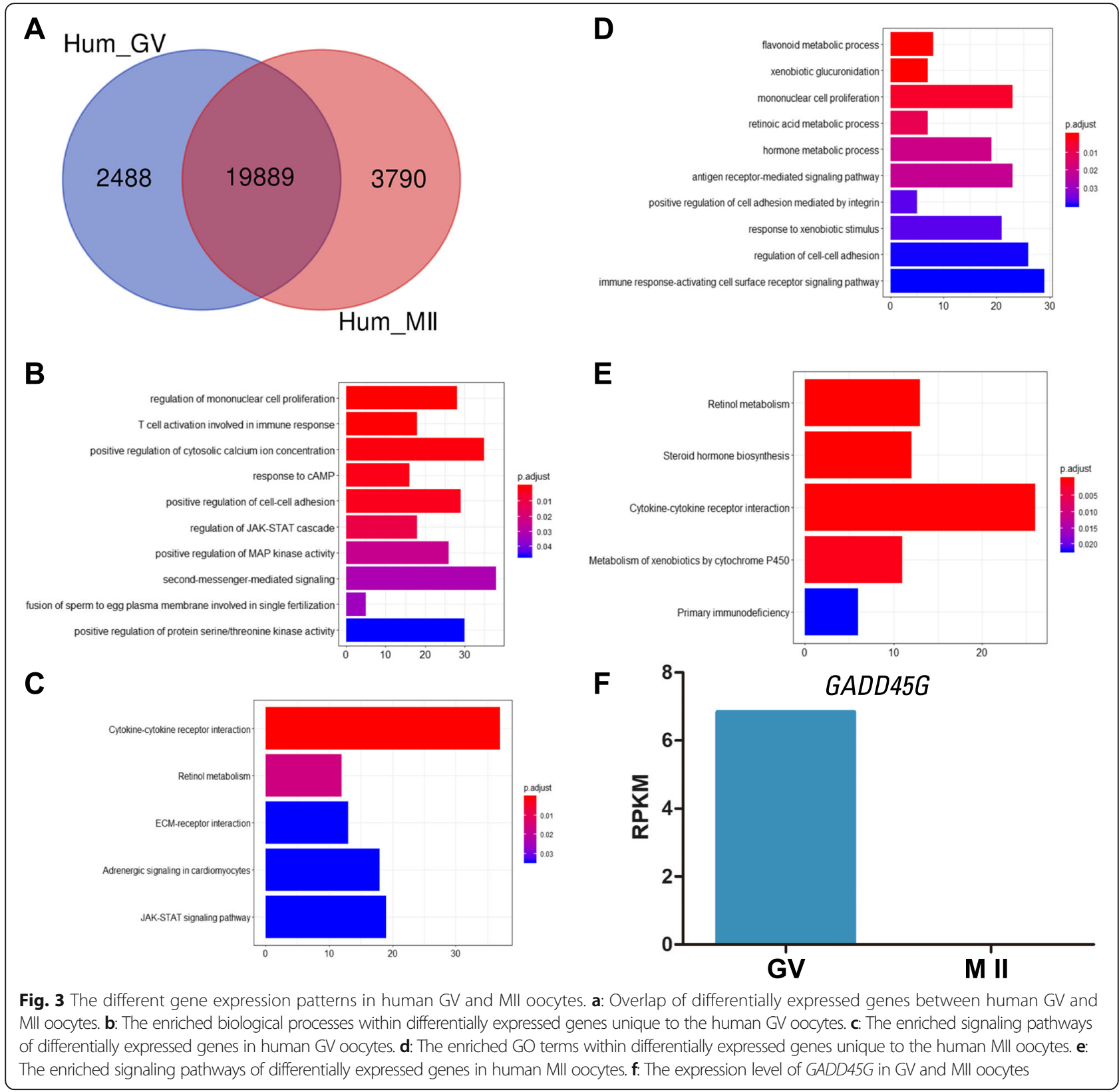

complete degradation of GADD45G during the GV-to-MII transition suggests its role in oocyte maturation (Fig. $3 f$ ).

\section{Comparison of gene expression profiles between human and mouse oocytes}

Oogenesis is a species specialized developmental process and the mouse is not the most suitable animal model for humans, especially when exploring oocyte maturation and fertilization [30,31]. To examine the differences in regulating oocyte maturation, we compared the gene expression profiles between human and mouse oocytes. A total of 9365 genes overlapped between human and mouse GV oocytes (Fig. 4a), of which only 120 transcripts specifically expressed in the GV oocytes overlapped in human and mouse (Fig. 4b). Furthermore, 8513 genes overlapped between human MII and mouse MII oocytes (Fig. 4c), of which only 19 transcripts that specially were expressed in MII oocytes overlapped in human and mouse (Fig. 4d). Collectively, these data suggest that there is a different regulatory network responsible for human and mouse oocyte maturation.

The ratio of non-homologous genes in human and mouse MIl oocytes

The ratio of non-homologous genes in oocytes could pinpoint the differences among species. Exclusively 
Table 2 Degradation of transcripts during human oocyte GV-toMIl transition

\begin{tabular}{|c|c|c|}
\hline Gene & GV RPKM & MII RPKM \\
\hline \multicolumn{3}{|c|}{ Translational termination } \\
\hline MRPL28 & 10.3237999 & 0.930954689 \\
\hline MRPS11 & 4.514520788 & 0.540265841 \\
\hline UPF1 & 2.066086591 & 1.165330501 \\
\hline MTRF1 & 3.654566571 & 1.87621318 \\
\hline MRPS22 & 8.353415364 & 0.713688366 \\
\hline \multicolumn{3}{|c|}{ Cytosolic transport } \\
\hline PIK3C3 & 3.50643722 & 1.239482851 \\
\hline STX5 & 3.076809772 & 0.299874951 \\
\hline GOSR1 & 3.701789336 & 1.399290475 \\
\hline WIPI1 & 2.162167036 & 1.177426334 \\
\hline ANKRD27 & 6.941615019 & 1.914800745 \\
\hline YKT6 & 4.572012219 & 0.565371648 \\
\hline GGA1 & 2.039797289 & 0.258461412 \\
\hline WDR91 & 2.82799633 & 0.248637966 \\
\hline SNF8 & 3.836114823 & 1.310913087 \\
\hline SYS1 & 4.181614332 & 1.120275234 \\
\hline \multicolumn{3}{|c|}{ Golgi vesicle transport } \\
\hline RNF139 & 7.365387964 & 1.444282184 \\
\hline CCDC22 & 5.252229072 & 0.649054735 \\
\hline TMED1 & 14.80813427 & 1.352350738 \\
\hline LMAN2L & 2.639099354 & 1.064585623 \\
\hline PREB & 9.939356968 & 1.762995138 \\
\hline \multicolumn{3}{|c|}{ Vesicle docking } \\
\hline VPS11 & 2.261760319 & 0.278176398 \\
\hline SNPH & 3.842344389 & 1.252070021 \\
\hline VPS33A & 2.495807447 & 0.291106366 \\
\hline$R A B 3 D$ & 2.191925935 & 1.123575285 \\
\hline STX10 & 9.092859067 & 1.0749016 \\
\hline RABEPK & 4.980868195 & 1.067827433 \\
\hline
\end{tabular}

expressed genes would discriminate the species-specific molecular pathways between human and mouse oocyte maturation. To further determine the ratio of nonhomologous genes in human and mouse MII oocytes, we downloaded the homologous gene database from NCBI (ftp://ftp.ncbi.nih.gov/pub/HomoloGene/). In human MII oocytes, 8834 genes are non-homologous genes, and the ratio of non-homologous genes is 37\% (Fig. 5a and b; Additional file 11). Whereas 1183 genes are non-homologous genes in mouse MII oocytes, and the ratio of non-homologous genes is $8 \%$ (Fig. $5 \mathrm{c}$ and d; Additional file 12).

To further explore the functions of non-homologous genes, we performed GO analysis on non-homologous genes in human and mouse MII oocytes. The enriched biological functions of human non-homologous genes are related to fertilization, such as "plasma membrane invagination", "sister chromatid cohesion" and "cell recognition" (Fig. 5e; Additional file 13). Interestingly, the "cAMP-mediated signaling" and "adenylate cyclaseactivating G protein-coupled receptor signaling pathway" are also enriched in human MII oocytes (Additional file 13). In contrast to the "response to cAMP" in human GV oocytes, all transcripts enriched in these two categories are Adhesion G Protein-Coupled Receptor (ADGR) gene families (Additional file 13), which may be involved in the regulation of calcium ion homeostasis in human MII oocytes [32]. However, we did not acquire the GO terms of non-homologous genes in mouse MII oocytes (data not shown). These results indicate that there is a significant difference between human and mouse MII oocytes.

\section{Discussion}

Female infertility and related reproductive disorders have overall health implications. The etiology of infertility remains elusive, and the exploration of etiology is predominantly based on studies of animal models. However, oogenesis is a species specialized process [31]. Although mouse is suited for studies of oocyte maturation and fertilization, the differences between mouse and human also need to be focused. Comparative analysis of RNA-Seq data has provided informative insights into the differences between human and mouse oocytes.

Oocyte maturation and fertilization have been explored for many years [21, 33, 34]; however, little is known regarding the different mechanisms between human and mouse. Here, we analyzed the transcriptomes of human and mouse GV and MII oocytes to explore the differences between human and mouse oocytes at the transcriptional level [22, 35]. As reported above, the regulation of oocyte maturation and fertilization exhibits different features in human and mouse oocytes. For example, compared to GV oocytes, the number of apparent transcripts in mouse and human MII oocytes are 430 and 3790, respectively (Figs. 1a and 3a). Noticeably, the number of transcripts may be slightly biased due to the limited number of samples studied and the different stages of functional annotation of the genomes. Additionally, differential gene expression analysis between human and mouse oocytes identified numerous significant differentially expressed genes that were enriched for typical or species-specific pathways and biological processes. The transcriptomes of human and mouse oocytes are highly variable, and the human oocytes exhibit more complexity than mouse oocytes.

Extensive degradation of transcripts occurs during oocyte maturation, which is required for oocyte to embryo transition. Several studies have explored the mechanisms 

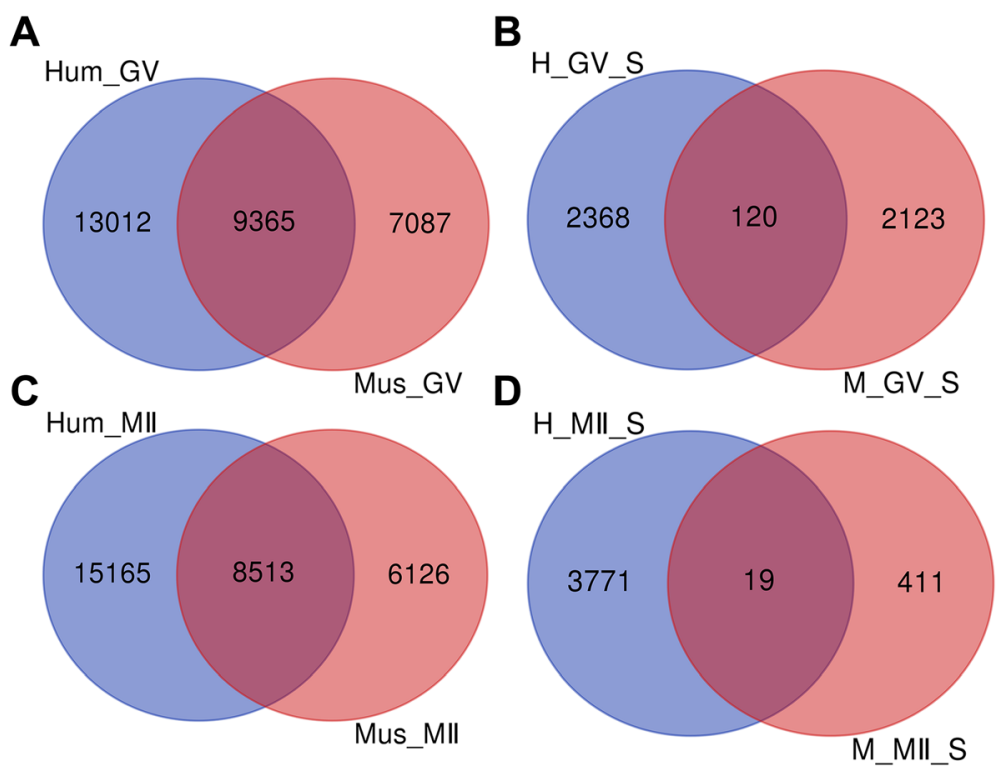

Fig. 4 The different gene expression patterns between human and mouse oocytes. a: Venn diagram shows overlapping of differentially expressed genes in human and mouse GV oocytes. b: Overlap of differentially expressed genes between human GV-specific genes and mouse GV-specific genes. c: Venn diagram shows overlapping of differentially expressed genes in human and mouse MIl oocytes. d: Overlap of differentially expressed genes between human MII-specific genes and mouse MII-specific genes

of maternal mRNA decay in mouse oocytes. For example, BTG4, CNOT6L and ZAR1/2 participate in the destruction of target specific transcripts during oocyte maturation $[13,34,36]$. However, the differences of selectively degraded transcripts between human and mouse have not been explored in detail. In this study, we have presented results showing that the number of completely degraded transcripts is similar (2243 and 2488 , respectively), but only 120 transcripts were overlapped. As expected, the majority of GO categories of these degraded transcripts between mouse and human oocytes are different. Therefore, the difference in selectively degraded transcripts between human and mouse oocytes suggests deviations of regulatory mechanisms that control oocyte maturation.

On the other hand, there are certain transcripts that appear to be up-regulated in MII oocytes. Moreover, the majority of GO terms of these transcripts between mouse and human oocytes are different except for some immunity-related categories. Although we didn't perform extra experiments to validate the results with human oocytes, several studies have reached some similar results, especially in the enriched signaling pathways at the corresponding stages [37-39]. As we all know, fullygrown GV oocytes are transcriptionally silent before resumption of meiosis until after fertilization when zygote genome activation occurs [8]. Therefore, the transcripts that exclusively appeared in MII oocytes may not be newly transcribed. And the reason of origin of these transcripts needs to be further explored.
The novelty and conservation of gene expression profiles shape the differences of species [3]. Two-level analysis of transcriptome demonstrated that the significant differences in molecular responses exist between human and mouse oocytes. The ratio of non-homologous genes in human MII oocytes is four times higher than that in mouse MII oocytes. However, most GO categories of non-homologous genes in human MII oocytes are conserved biological processes including "adenylate cyclase-activating G proteincoupled receptor signaling pathway" and "cAMP-mediated signaling". Unexpectedly, we hardly detected any GO terms in non-homologous genes of mouse MII oocytes. Therefore, the strong evidence for diversification of nonhomologous genes prompts us to hypothesize that the functions of novel loci expressed in the oocytes are shaped by the forces of gametic selection that target the processes unique to individual species. Therefore, it shows potential shortcomings related to the use of mouse model to explore the oocyte maturation process in human.

\section{Conclusions}

In summary, human and mouse oocytes exhibit divergent transcriptomes at the fully-grown GV and MII stages, probably deciphering the differential molecular response to oocyte maturation and fertilization. Critical factors involved in oocyte maturation were found to be differentially expressed between human and mouse oocytes. Moreover, human MII oocytes exhibited a higher ratio of non-homologous genes compared to mouse MII oocytes, which were enriched for various biological 

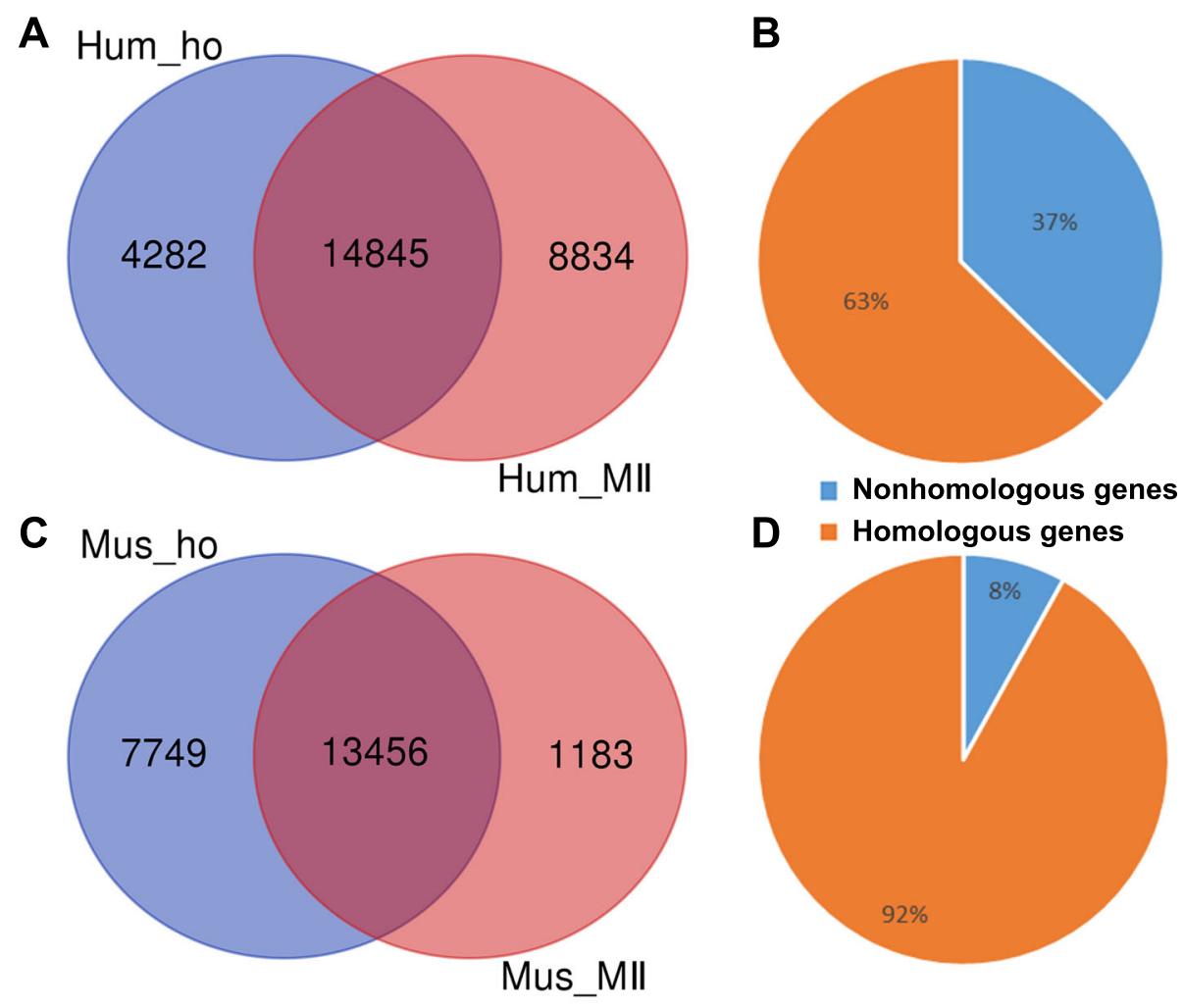

D Homologous genes

$\mathbf{E}$

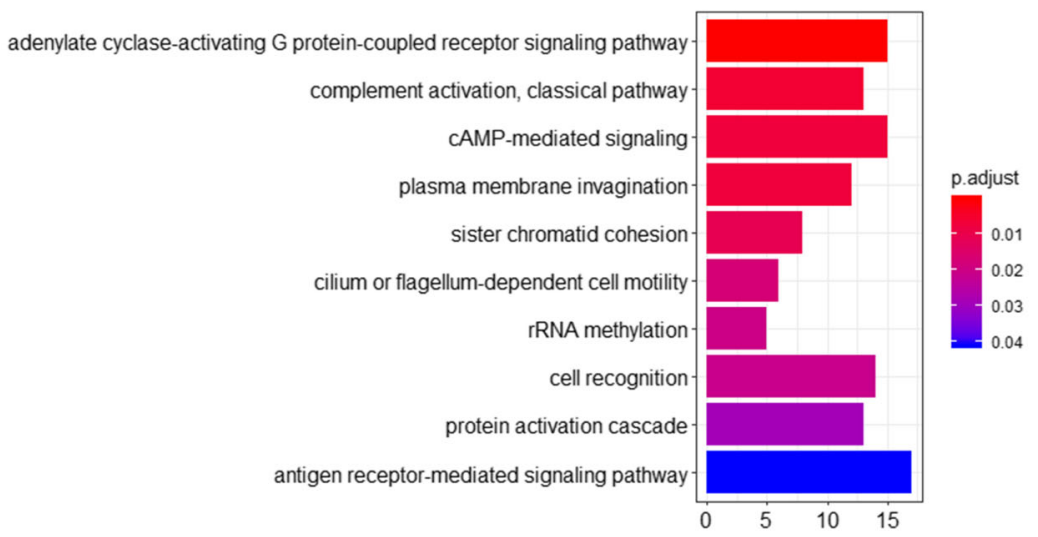

Fig. 5 The difference of non-homologous genes between human and mouse Mll oocytes. a: Venn diagram shows overlapping genes in human homologous genes database and human MII expressed genes. $\mathbf{b}$ : The ratio of non-homologous genes in human MII oocytes. c: Venn diagram shows overlapping genes in mouse homologous genes database and mouse Mll expressed genes. $\mathbf{d}$ : The ratio of nonhomologous genes in mouse MII oocytes. e: The enriched biological processes within non-homologous genes unique to the human MII oocytes

processes that play important roles during oocyte maturation. These findings show significant differences in gene expression profiles between human and mouse oocytes, limiting the generalizations from mouse to human oocyte maturation. Knowledge about the limitations of animal models is crucial when exploring a complex process such as human oocyte maturation and fertilization.

\section{Methods}

Comparation of library preparation and data processing Mouse (C57BL/6 N) oocyte and early embryo transcriptome data were obtained from Zhang et al. [22], who used Smart-Seq2 protocol with slightly modification to prepare the RNA-Seq libraries [40]. All cDNA libraries were fragmented and then sequenced on Illumina HiSeq platform. After sequencing, the raw reads in the fastq 
format were first processed and the clean reads were obtained by removing adapter sequences and low-quality reads from raw data. Next, the clean reads were aligned to the reference genome (mm9) using TopHat (version 2.0.11). Based on the comparison results, the sequencing data were assembled by Cufflinks (version 2.0.2). And the normalization of the gene expression values was based on the fragments per kilobase of exon per million reads mapped (FPKM).

Human oocyte transcriptome data were obtained from Reyes et al. [35]. The total RNA of GV and MII human oocytes were purified according to the manufactures' protocol, which were then amplified using SMARTer Ultra Low Input RNA HV kit (Clontech, USA). The cDNA libraries were fragmented and then sequenced on a paired-end $2 \times 100 \mathrm{bp}$ Illumina Hiseq 2500 platform. After sequencing, the raw reads were processed using CLC Genomics Workbench (CLC, v7.5.1; Qiagen, USA) to remove the Clontech IS PCR primer (5' - AAGCAG TGGTATCAACGCAGAGTAC-3') from the raw data. The clean reads were then aligned to the annotated human genome (GRCh38.82) according to the RNA-Seq tool instruction with small modification. CLC normalized expression values for each gene as total exon reads per kilobase of transcript per million mapped reads (RPKM). Differential expression analysis was performed using the DESeq2 package (v1.10.1) through mean normalized exon reads that exported from CLC Genomics Workbench.

Different library preparation and data processing procedures could lead to the bias of detected genes and gene expression levels. To reduce the bias of data, we selected all the transcripts, whose expression levels $>0$ in this study.

\section{Expression analysis of transcriptomes}

Normalized FPKM and RPKM values for mouse and human oocyte maturation and fertilization stages were obtained from GEO database (GSE71434_FPKM_stage.txt.gz; GSE95477_Differentially_Expressed_Genes_and_RPKM_ Values.csv.gz). Data from the following stages were used in this study: 8-week GV oocytes, MII oocytes, zygotes and 2cell embryos from Bing-Jie Zhang et al. [22] and young women $(<30$ years) GV and MII oocytes from Reyes et al. [35]. The transcripts with average FPKM or RPKM $>0$ at all above stages were retained for further analysis. To identify the genes uniquely expressed in a given group, we compared the two groups of samples through Draw Venn Diagram (http://bioinformatics.psb.ugent.be/ webtools/Venn/). In addition, homologous gene database 'build68' was downloaded (ftp://ftp.ncbi.nih.gov/ pub/HomoloGene/) to calculate the ratios of the nonhomologous genes in mouse and human MII oocytes.

\section{Gene ontology and KEGG analysis}

Gene ontology and KEGG analysis was performed by using the ClusterProfiler R package [19]. Given a list of genes, symbol gene IDs were translated into entre IDs through bitr function. The GO and KEGG analysis were conducted through enrich GO and enrich KEGG functions, respectively. The analysis of the enrichment of uniquely expressed genes was conducted and a corrected $P$-value $\leq 0.05$ was considered to indicate significant gene enrichment.

\section{Supplementary information}

Supplementary information accompanies this paper at https://doi.org/10. 1186/s12864-020-06885-4.

Additional file 1. Mouse GV oocyte-specific expressed gene list. Additional file 2. Mouse MII oocyte-specific expressed gene list. Additional file 3. GO categories of mouse GV oocyte-specific expressed genes.

Additional file 4. GO categories of mouse MII oocyte-specific expressed genes.

Additional file 5. Human GV oocyte-specific expressed gene list.

Additional file 6. Human MII oocyte-specific expressed gene list.

Additional file 7. GO categories of human GV oocyte-specific expressed genes.

Additional file 8. KEGG categories of human GV oocyte-specific expressed genes.

Additional file 9. GO categories of human MII oocyte-specific expressed genes.

Additional file 10. KEGG categories of human MII oocyte-specific expressed genes.

Additional file 11. Human MII oocyte non-homologous gene list. Additional file 12. Mouse MII oocyte non-homologous gene list. Additional file 13. GO categories of human MII oocyte nonhomologous genes.

\begin{abstract}
Abbreviations
BP: Biological processes; FPKM: Fragments per kilobase of exon per million reads mapped; GADD45G: Growth arrest and DNA damage 45G; GEO: Gene expression omnibus; GO: Gene ontology; IVF: In vitro fertilization; KEGG: Kyoto encyclopedia of genes and genomes; MAPK: Mitogen-activated protein kinase; RPKM: Reads per kilobase of exon per million reads mapped
\end{abstract}

\section{Acknowledgements}

We appreciate help from members of the Sun laboratory for comments during preparation of the manuscript.

\section{Authors' contributions}

QYS and ZHZ designed the project. ZHZ performed the data analysis. TGM and $A L$ analyzed the transcriptome data. QYS and ZHZ wrote the paper. QYS, $\mathrm{HS}$ and ZBW edited the manuscript. All authors read and approved the final manuscript.

\section{Funding}

This work was supported by the National R\&D Program of China (2018YFA0107701) and the National Natural Science Foundation of China (31801245). The funding bodies played no role in the design of the study and collection, analysis, and interpretation of data and in writing the manuscript. 


\section{Availability of data and materials}

The data sets analyzed here are publicly available. The mouse oocyte data set is available on the Gene Expression Omnibus (GEO) database, and the accession number is GSE71434, and the human oocyte data set is available on the GEO database, and the accession number is GSE95477. The reference genome (mm9) was downloaded from the website (http://hgdownload.cse. ucsc.edu/goldenPath/mm9/chromosomes/). The annotated human genome (GRCh38.82) was downloaded from the website (https://www.ncbi.nlm.nih. gov/genome/guide/human/).

\section{Ethics approval and consent to participate}

Patients undergoing infertility treatment via IVF, including the use of donor oocytes, at the Colorado Center for Reproductive Medicine (Lone Tree, CO, USA) provided informed consent that materials not used and normally discarded during the IVF cycle could be used for research. The informed consent obtained was written and approved by the Western Institutional Review Board (IRB\#1151520).

\section{Consent for publication}

Not Applicable.

\section{Competing interests}

The authors declare that they have no competing interests.

\section{Author details}

${ }^{1}$ State Key Laboratory of Stem Cell and Reproductive Biology, Institute of Zoology, Chinese Academy of Sciences, Beijing, China. ${ }^{2}$ University of Chinese Academy of Sciences, Beijing, China. ${ }^{3}$ Fertility Preservation Lab, Reproductive Medicine Center, Guangdong Second Provincial General Hospital, Guangzhou 510317, China. ${ }^{4}$ Department of Veterinary Pathobiology, University of Missouri, Columbia, MO 65211, USA.

Received: 24 February 2020 Accepted: 6 July 2020 Published online: 10 July 2020

\section{References}

1. Wassarman PM, Litscher ES. Influence of the zona pellucida of the mouse egg on folliculogenesis and fertility. Int J Dev Biol. 2012;56(10-12):833-9.

2. Baerwald AR, Adams GP, Pierson RA. Ovarian antral folliculogenesis during the human menstrual cycle: a review. Hum Reprod Update. 2012;18(1):73-91.

3. Evsikov AV, Graber JH, Brockman JM, Hampl A, Holbrook AE, Singh P, Eppig JJ, Solter D, Knowles BB. Cracking the egg: molecular dynamics and evolutionary aspects of the transition from the fully grown oocyte to embryo. Genes Dev. 2006;20(19):2713-27.

4. Cha KY, Chian RC. Maturation in vitro of immature human oocytes for clinical use. Hum Reprod Update. 1998;4(2):103-20.

5. Schultz RM, Wassarman PM. Biochemical studies of mammalian oogenesis: protein synthesis during oocyte growth and meiotic maturation in the mouse. J Cell Sci. 1977;24:167-94.

6. Stricker SA, Cline C, Goodrich D. Oocyte maturation and fertilization in marine nemertean worms: using similar sorts of signaling pathways as in mammals, but often with differing results. Biol Bull. 2013;224(3):137-55.

7. Gong S, Sun GY, Zhang M, Yuan HJ, Zhu S, Jiao GZ, Luo MJ, Tan JH. Mechanisms for the species difference between mouse and pig oocytes in their sensitivity to glucorticoids. Biol Reprod. 2017;96(5):1019-30.

8. Telford NA, Watson AJ, Schultz GA. Transition from maternal to embryonic control in early mammalian development: a comparison of several species. Mol Reprod Dev. 1990;26(1):90-100

9. Bouniol-Baly C, Hamraoui L, Guibert J, Beaujean N, Szollosi MS, Debey P. Differential transcriptional activity associated with chromatin configuration in fully grown mouse germinal vesicle oocytes. Biol Reprod. 1999;60(3):580-7.

10. De La Fuente R. Chromatin modifications in the germinal vesicle (GV) of mammalian oocytes. Dev Biol. 2006;292(1):1-12.

11. Schultz RM. Regulation of zygotic gene activation in the mouse. BioEssays. 1993;15(8):531-8.

12. Su YQ, Sugiura K, Woo Y, Wigglesworth K, Kamdar S, Affourtit J, Eppig JJ. Selective degradation of transcripts during meiotic maturation of mouse oocytes. Dev Biol. 2007;302(1):104-17.

13. Sha QQ, Yu JL, Guo JX, Dai XX, Jiang JC, Zhang YL. CNOT6L couples the selective degradation of maternal transcripts to meiotic cell cycle progression in mouse oocyte. EMBO J. 2018;37(24):e99333.
14. Barckmann B, Simonelig M. Control of maternal mRNA stability in germ cells and early embryos. Biochim Biophys Acta. 2013;1829(6-7):714-24.

15. Ma J, Fukuda Y, Schultz RM. Mobilization of dormant Cnot7 mRNA promotes Deadenylation of maternal transcripts during mouse oocyte maturation. Biol Reprod. 2015;93(2):48.

16. Madissoon E, Tohonen V, Vesterlund L, Katayama S, Unneberg P, Inzunza J, Hovatta $\mathrm{O}$, Kere J. Differences in gene expression between mouse and human for dynamically regulated genes in early embryo. PLoS One. 2014; 9(8):e102949.

17. Kocabas AM, Crosby J, Ross PJ, Otu HH, Beyhan Z, Can H, Tam WL, Rosa GJ, Halgren RG, Lim B, et al. The transcriptome of human oocytes. Proc Natl Acad Sci U S A. 2006;103(38):14027-32.

18. Biase FH. Oocyte developmental competence: insights from cross-species differential gene expression and human oocyte-specific functional gene networks. Omics. 2017:21(3):156-68.

19. Yu G, Wang LG, Han Y, He QY. ClusterProfiler: an R package for comparing biological themes among gene clusters. Omics. 2012;16(5):284-7.

20. Sheng JJ, Jin JP. TNNI1, TNNI2 and TNNI3: evolution, regulation, and protein structure-function relationships. Gene. 2016:576(1 Pt 3):385-94.

21. Satouh $Y$, Ikawa M. New insights into the molecular events of mammalian fertilization. Trends Biochem Sci. 2018;43(10):818-28.

22. Zhang B, Zheng H, Huang B, Li W, Xiang Y, Peng X, Ming J, Wu X, Zhang Y, $\mathrm{Xu} Q$, et al. Allelic reprogramming of the histone modification H3K4me3 in early mammalian development. Nature. 2016;537(7621):553-7.

23. Bianchi E, Wright GJ. Sperm meets egg: the genetics of mammalian fertilization. Annu Rev Genet. 2016;50:93-111.

24. Kinsey $\mathrm{WH}$. Intersecting roles of protein tyrosine kinase and calcium signaling during fertilization. Cell Calcium. 2013;53(1):32-40.

25. Aquino-Martinez R, Artigas N, Gamez B, Rosa JL, Ventura F. Extracellular calcium promotes bone formation from bone marrow mesenchymal stem cells by amplifying the effects of BMP-2 on SMAD signalling. PloS One. 2017:12(5):e0178158.

26. Freter RR, Schultz RM. Regulation of murine oocyte meiosis: evidence for a gonadotropin-induced, cAMP-dependent reduction in a maturation inhibitor. J Cell Biol. 1984;98(3):1119-28.

27. Liu C, Zhang W, Yang D, Liu Y. Molecular characterization, polymorphism, and Association of Porcine GADD45G gene. Anim Biotechnol. 2015;26(3):230-6.

28. Johnen H, Gonzalez-Silva L, Carramolino L, Flores JM, Torres M, Salvador JM. Gadd45g is essential for primary sex determination, male fertility and testis development. PLoS One. 2013;8(3):e58751.

29. Gierl MS, Gruhn WH, von Seggern A, Maltry N, Niehrs C. GADD45G functions in male sex determination by promoting p38 signaling and Sry expression. Dev Cell. 2012;23(5):1032-42.

30. Menezo YJ, Herubel F. Mouse and bovine models for human IVF. Reprod BioMed Online. 2002:4(2):170-5.

31. Santos RR, Schoevers EJ, Roelen BA. Usefulness of bovine and porcine IVM/ IVF models for reproductive toxicology. Reprod Biol Endocrinol. 2014;12:117.

32. Orsini CA, Setlow B, DeJesus M, Galaviz S, Loesch K, loerger T, Wallis D. Behavioral and transcriptomic profiling of mice null for Lphn3, a gene implicated in ADHD and addiction. Mol Genet Genom Med. 2016;4(3):322-43.

33. Yang Y, Yang CR, Han SJ, Daldello EM, Cho A, Martins JPS, Xia G, Conti M. Maternal mRNAs with distinct 3' UTRs define the temporal pattern of Ccnb1 synthesis during mouse oocyte meiotic maturation. Genes Dev. 2017;31(13): 1302-7.

34. Rong Y, Ji SY, Zhu YZ, Wu YW, Shen L, Fan HY. ZAR1 and ZAR2 are required for oocyte meiotic maturation by regulating the maternal transcriptome and mRNA translational activation. Nucleic Acids Res. 2019;47(21):11387-402.

35. Reyes JM, Silva E, Chitwood JL, Schoolcraft WB, Krisher RL, Ross PJ. Differing molecular response of young and advanced maternal age human oocytes to IVM. Hum Reprod. 2017;32(11):2199-208.

36. Sha QQ, Dai XX, Dang Y, Tang F, Liu J, Zhang YL, Fan HY. A MAPK cascade couples maternal mRNA translation and degradation to meiotic cell cycle progression in mouse oocytes. Development. 2017;144(3):452-63.

37. Barragán M, Pons J, Ferrer-Vaquer A, Cornet-Bartolomé D, Schweitzer A, Hubbard J, Auer H, Rodolosse A, Vassena R. The transcriptome of human oocytes is related to age and ovarian reserve. Mol Hum Reprod. 2017;23(8): 535-48.

38. Grøndahl ML, Borup R, Vikeså J, Ernst E, Andersen CY, Lykke-Hartmann K. The dormant and the fully competent oocyte: comparing the transcriptome of human oocytes from primordial follicles and in metaphase II. Mol Hum Reprod. 2013;19(9):600-17. 
39. Zhang Y, Yan Z, Qin Q, Nisenblat V, Chang HM, Yu Y, Wang T, Lu C, Yang $M$, Yang $S$, et al. Transcriptome landscape of human folliculogenesis reveals oocyte and granulosa cell interactions. Mol Cell. 2018;72(6):10211034.e1024.

40. Picelli S, Faridani OR, Björklund AK, Winberg G, Sagasser S, Sandberg R. Fulllength RNA-seq from single cells using smart-seq2. Nat Protoc. 2014;9(1): $171-81$.

\section{Publisher's Note}

Springer Nature remains neutral with regard to jurisdictional claims in published maps and institutional affiliations.

Ready to submit your research? Choose BMC and benefit from:

- fast, convenient online submission

- thorough peer review by experienced researchers in your field

- rapid publication on acceptance

- support for research data, including large and complex data types

- gold Open Access which fosters wider collaboration and increased citations

- maximum visibility for your research: over $100 \mathrm{M}$ website views per year

At $\mathrm{BMC}$, research is always in progress.

Learn more biomedcentral.com/submissions 